\title{
THE INCREASED PLASMA VOLUME IN CARDIAC INSUFFICIENCY: ITS CORRELATION WITH RIGHT-SIDED FAILURE
}

\author{
By GEORGE A. PERERA \\ (From the Department of Medicine, College of Physicians and Surgeons, Columbia \\ University, and the Presbyterian Hospital, New York City)
}

(Received for publication March 6, 1945)

It is now well established that in,cardiac insufficiency there is an increase in the circulating blood volume as calculated by dye methods $(1,2)$. The mechanism of the rise in the red cell component has been attributed to anoxemia (3); that of the increase in plasma volume has remained obscure.

Discrepancies in the different quantitative measurements of cardiac insufficiency are not rare. The occasional appearance of acute right-sided failure without a comparable rise in peripheral venous pressure has been observed in this hospital. In addition, others have noted that the plasma volume rise preceded the venous pressure change in several cardiac patients in whom failure was induced by sodium chloride administration (4). Although a general parallelism between the blood volume, circulation time and venous pressure has been reported (1), other workers were unable to determine any significant relationship between these values (2).

Because of the lack of consistent correlation between the various clinical measurements of cardiac insufficiency, the present study was undertaken to determine whether the plasma volume might be related to the degree of circulatory interference in the hepatic and portal vascular beds. This was based on the hypothesis that these areas were larger and more logical sites for the volume increase than the smaller pulmonary and more erratic peripheral circulations.

\section{MATERIAL AND METHODS}

Sixteen patients with heart disease were studied on the wards of the Presbyterian Hospital, all having been admitted because of uncomplicated acute cardiac insufficiency and with no evidence of acute or chronic blood loss or shock. Only those patients whose heart failure was predominantly right (portal and systemic congestion) or left-sided (pulmonary congestion) were included, and they were divided into 2 equal groups on this basis. Although several of each group gave a history of previous episodes of cardiac insufficiency, the duration of acute signs or symptoms was not longer than 2 weeks before admission in any instance. Every effort was made to secure data as soon after hospital admission as possible and before essential therapy had produced any modification of the clinical picture. The average age of the 2 groups of patients was comparable, although they varied in age from the fourth to the seventh decade.

All observations were made within a period of several hours. Blood samples were obtained with the patient lying in bed with a $30^{\circ}$ head gatch, and after at least a 20-minute period of inactivity in that position. Circulation times were measured with either sodium dehydrocholate or calcium gluconate. The plasma volume was determined with the blue dye T-1824, the optical density being measured with the photoelectric colorimeter (5). Multiple serum samples were obtained after 10 minutes because of expected variations and delay in mixing. Predictions of plasma volumes were based on the data of Gibson and Evans (6) and the observed hematocrit, using both weight and surface area for comparison. The difference between observed and predicted values was expressed as a percentage deviation from normal. Determinations of red cell volume were not made because of the variable and conflicting results obtained by current methods.

\section{RESULTS}

The results are shown in tabular form. It is apparent that the patients with right-sided cardiac insufficiency exhibited a greater increase in plasma volume than did those with left-sided failure. Although the degree of insufficiency was in general reflected in other measurements, such as venous pressure, circulation time, and vital capacity, only a rough parallelism between these values and the plasma volume was observed. Quantitative correlation with liver size was impossible because of difficulties in accurate measurements, because of variations in body build and the position of the diaphragms, and because antecedent liver enlargement could not be excluded. Nevertheless, the rise in plasma volume was also generally related to the degree of liver enlargement (Figure 1).

Predicted plasma volumes based on weight are regarded as unsatisfactory in cardiac patients because of edema. Yet, at times, the comparison between predicted values based on height and surface area showed marked deviation from each 


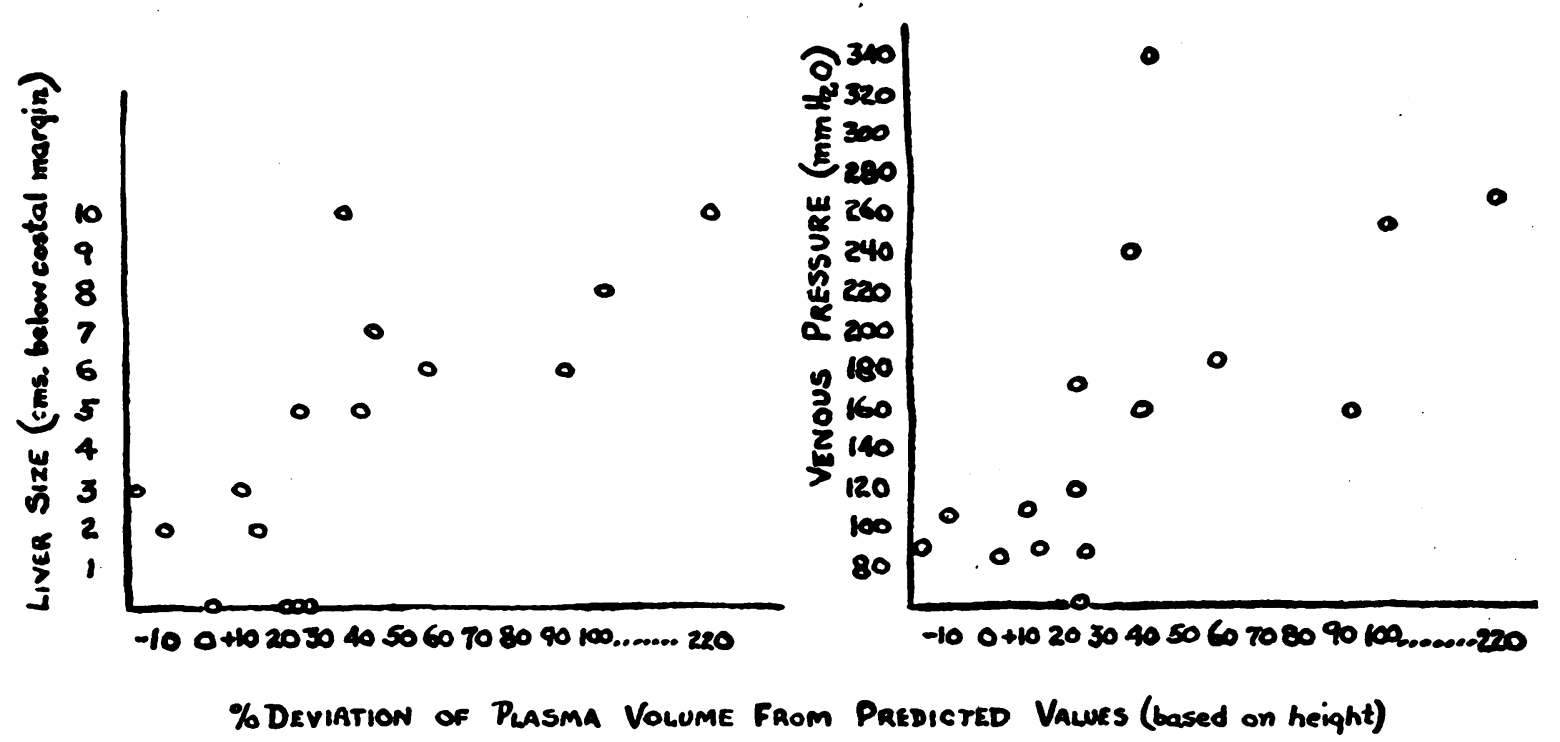

Fig. 1. The Relationship of the Plasma Volume to Liver Size and Venous Pressure

other in either direction. This suggests that any single method of estimating normal values may sometimes be subject to error and that only major changes in plasma volume can be regarded as significant in a study of this kind.

\section{DISCUSSION}

The observation that the increase in plasma volume may be correlated with right-sided cardiac insufficiency is in itself neither surprising nor illogical. Left-sided failure does not permit large volume increases as it requires but small reductions in vital capacity to produce marked symptoms; whereas major increases in congestion in the portal and peripheral bed can be tolerated readily. At autopsy, the liver in cardiac insufficiency may be greatly increased in weight, its vascular channels dilated and congested, and a likely reservoir for many hundred $\mathrm{ml}$. of blood.

The increase in plasma volume noted in this study cannot be explained by loss of dye in edema fluid (1). Although the initial rapid fall in concentration of the dye is delayed in cardiac insufficiency, presumably because of delayed mixing, no positive evidence could be obtained to show that there was any abnormal loss of dye from the circulation. Comparison of the subsequent constant disappearance slope in the patients with increased volumes gave no indication of a faster removal of dye than existed in those with left-sided failure.
As regards the mechanism of the increase in plasma volume in right-sided cardiac insufficiency, it cannot be explained solely by the retention of salt and water. Oliguria or anuria due to other than cardiac failure is not accompanied by changes in plasma volume of the same degree or rapidity as seen in heart failure. It has been suggested that in cardiac insufficiency the kidneys may not be able to excrete salt and water in a normal manner and that this may be a function of decreased cardiac output rather than secondary to congestion (4). The observation that certain patients first gain weight, then increase blood volume, and only later demonstrate a rise in venous pressure, can only be explained by postulating a reduction in renal excretion secondary to heart failure.

There are several reasons which support the hypothesis that the portal and peripheral circulatory beds do not react similarly to the same increased load and that, as reviewed years ago by Krogh, the liver may have potentialities as a variable reservoir (7). These include the absence of any exact linear relationship between the plasma volume and the venous pressure or circulation time, as noted by others (2) and confirmed in this study. When excessive fluids are administered rapidly by vein, using the technique of Caughey (8), it is at times possible in both normal and cardiac patients to see enlargement of 


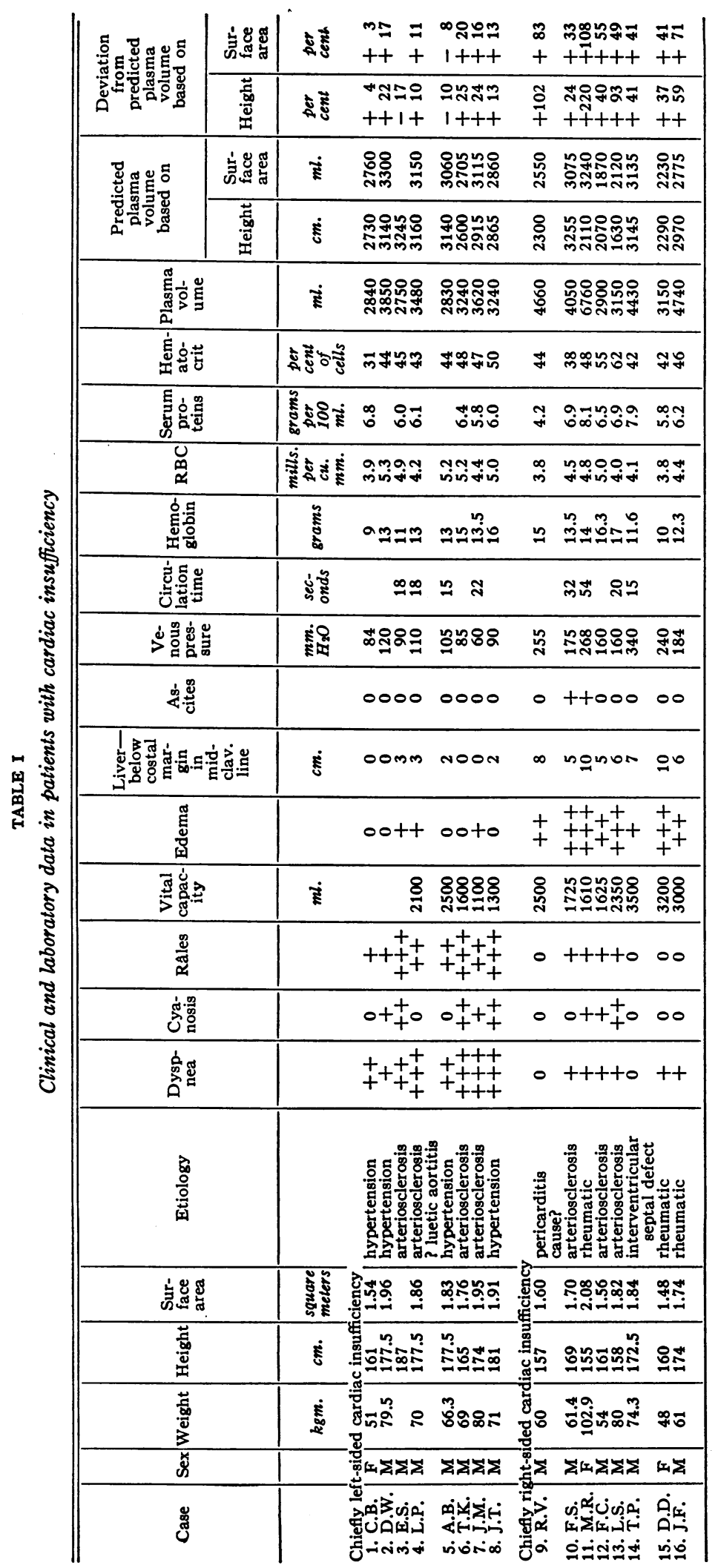


the liver without a comparable rise in venous pressure. The clinical observation that pressure on an enlarged liver in cardiac insufficiency produces a rise in venous pressure has been made repeatedly.

It is therefore suggested that the liver and possibly the entire portal circulation react to increases in hepatic vein pressure by dilatation and an enlarged venous bed. Peripheral veins on the other hand, perhaps because of better venous tone or increased pressure from without, respond to the same stimulus primarily by a rise in pressure and with less change in volume. A mechanism such as this may be at least a partial factor to account for the increased plasma volume in cardiac insufficiency.

\section{CONCLUSIONS}

1. The increase in plasma volume, frequently encountered in patients with cardiac insufficiency, appears to be associated with right-sided failure.

2. It is suggested that this increase is due to dilatation and engorgement of vascular channels in the liver and portal circulatory beds.

I am indebted to Miss Barbara Wright for assistance in compiling some of the clinical data, and to Dr. Dickinson W. Richards, Jr., for his valuable suggestions.

\section{BIBLIOGRAPHY}

1. Gibson, J. G., 2nd, and Evans, W. A., Jr., Clinical studies of the blood volume. III. Changes in blood volume, venous pressure and blood velocity rate in chronic congestive heart failure. J. Clin. Invest., 1937, 16, 851.

2. Meneely, G. R., and Kaltreider, N. L., A study of the volume of the blood in congestive heart failure. Relation to other measurements in fifteen patients. J. Clin. Invest., 1943, 22, 521.

3. Harrop, G. A., and Wintrobe, M. M., Polycythemia. In Handbook of Hematology, H. Downey, Editor, P. B. Hoeber, Inc., New York, 1938.

4. Warren, J. V.; and Stead, E. A., Jr., Fluid dynamics in chronic congestive heart failure. An interpretation of the mechanisms producing the edema, increased plasma volume and elevated venous pressure in certain patients with prolonged congestive failure. Arch. Int. Med., 1944, 73, 138.

5. Gibson, J. G., 2nd, and Evelyn, K. A., Clinical studies of the blood volume. IV. Adaptation of the method to the photoelectric microcolorimeter. J. Clin. Invest., 1938, 17, 153.

6. Gibson, J. G., 2nd, and Evans, W. A., Jr., Clinical studies of the blood volume. II. The relation of plasma and total blood volume to venous pressure, blood velocity rate, physical measurements, age and sex in ninety normal humans. J. Clin. Invest., 1937, 16, 317.

7. Krogh, A., The regulation of the supply of blood to the right heart. Skand. Arch. für Physiol., 1912, 27, 227.

8. Caughey, J. L., Jr., Effect of rapid infusion on venous pressure: a test of cardiac reserve. Proc. Soc. Exper. Biol. and Med., 1935, 32, 973. 